\title{
Determination of the Factors Controlling Crystallography Non-Conformance in Single Crystal Turbine Blade Production on an Industrial Scale
}

\author{
J. Cameron ${ }^{1, a}$, P.W. Shelton ${ }^{2, b}$ \\ ${ }^{1} \mathrm{~J}$. Cameron, PhD student, SST, University of Teesside \& VIM Metallurgist, FRS Ltd. \\ ${ }^{2}$ Dr. P.W. Shelton, Principal Lecturer, SST, University of Teesside \\ a ${ }^{\text {jcameron@firthrixson.com, }}$, p.w.Shelton@tees.ac.uk
}

Keywords: Single Crystal, Directional Solidification, Investment Casting, Crystallography, XRD

\begin{abstract}
The arduous conditions to which hot section turbine components are subjected in service, dictate the superior physical and mechanical properties demanded of them. The demand for both high temperature and creep resistance, and anisotropic property requirements of the components has lead to developments in alloy composition, component geometry and single, oriented grain structure design. The slim tolerances and high quality standards imposed on such design features, combined with the high production volume in industry means that component non-conformances to the customer specifications occur. The input variables contributing to crystallography nonconformance in single crystal production have been investigated with a view to defining optimum process parameters for the successful manufacture of single crystal investment cast components on an industrial scale.

\section{Introduction}

Towards the end of World War II, a demand for military jet engines with higher thrust levels fuelled the development of the Nickel-base Superalloys and the development of Single Crystal grain structures. Currently, gas inlet temperatures close to $1600^{\circ} \mathrm{C}$ and centrifugal forces approaching $200 \mathrm{MPa}^{[1]}$ demand properties in the turbine blades including high tensile strength and stiffness, with resistance to thermal shock, thermal fatigue, and an extended creep rupture life. Turbine section components are required to operate at temperatures approximating $0.8 \mathrm{Tm}$ (melting point of alloy). Despite demanding product tolerances, requiring narrow manufacturing process windows, the assumption would be, that given appropriate control of casting conditions, $100 \%$ yields for single crystal casting would be obtained. In real manufacturing situations however, this is not achieved ${ }^{[2]}$. Wagner describes industrial reject rates of up to $20 \%$ on such components, including significant non-conformances of crystallographic orientation. This paper reports an investigation into the influence of process variables thought to be responsible for the crystallographic non-conformance levels found in the production of Single Crystal Superalloy components.
\end{abstract}

\section{Crystallographic Structure}

The non-uniform, centripetal loading of functioning blades, acts parallel to the aerofoil length calling for anisotropic property requirements within the blade. It was recognized by VerSnyder et $a l^{[3]}$ that fracture of polycrystalline materials at high temperatures was generally initiated at grain boundaries lying transverse to the applied stress. It was reasoned that if the density of transverse boundaries could be reduced or even eliminated by producing wholly columnar-grained or single crystal components then the fracture process should be suppressed and a five fold improvement in the creep strength of the components might be achieved $^{[4]}$. Directionally solidified multi-crystalline castings were developed by Pratt and Whitney ${ }^{[5]}$ in the early 1960 s, with a non-random distribution of individual grains. The achievement of anisotropic crystalline texture encouraged trials of single crystal components in $1970^{[6]}$. Further control over grain orientation proved useful when anisotropic properties were aligned parallel to the load direction, the optimum orientation being that of the $<001>$ 
plane $^{[6]}$, the $<111>$ oriented components possessing superior properties yet not as readily produced as $<001>$ oriented components. Controlled crystallographic orientation of cast components is made feasible by the fact that Nickel-base Superalloys possess a face centred cubic lattice and that the preferred growth direction on solidification is parallel to the $<001>$ reference plane ${ }^{[7]}$. The desired crystal orientation may be achieved by the provision of a temperature gradient in the molten alloy, in the direction of the desired orientation, giving unidirectional heat loss in a direction parallel to that of the desired growth.

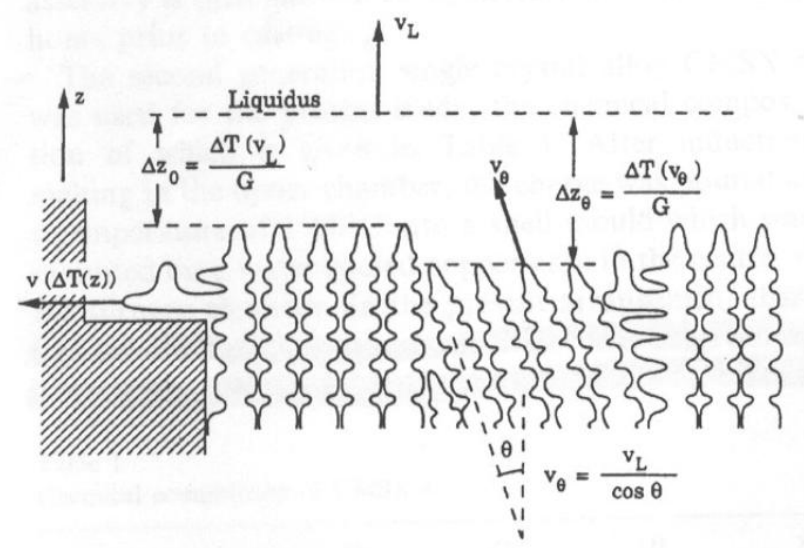

Figure 1 - Schematic representation of the 'pinching off' of less favourably oriented grains The possibility of crystallographic orientation control in a Single Crystal casting is dependent directly upon the competitive growth of dendrites along a temperature gradient. It has been proven $^{[8]}$ that the dendritic tip growth rate $\mathrm{v}$, depends upon the local 'tip' undercooling $\Delta \mathrm{T}_{\text {tip, }}$ below the liquidus temperature. The most plausible model for the relationship between $\mathrm{v}$ and $\Delta \mathrm{T}_{\text {tip }}$ is that proposed by Burden and Hunt ${ }^{[9]}$ in which the dendrite growth velocity $\mathrm{v}$, increases monotonically with the square of the undercooling, i.e. $\mathrm{v} \propto \Delta \mathrm{T}^{2}$ tip. Dendrites aligned with respect to the maximum gradient of the temperature field, must grow at a faster speed than those less favourably aligned. This effect allows those grains more favourably aligned to 'pinch off' the less well-aligned grains as depicted in Figure 1 resulting in a columnar grain structure with crystal orientations reflecting the imposed temperature gradient.

\section{Quality Control and Crystal Orientation Measurement}
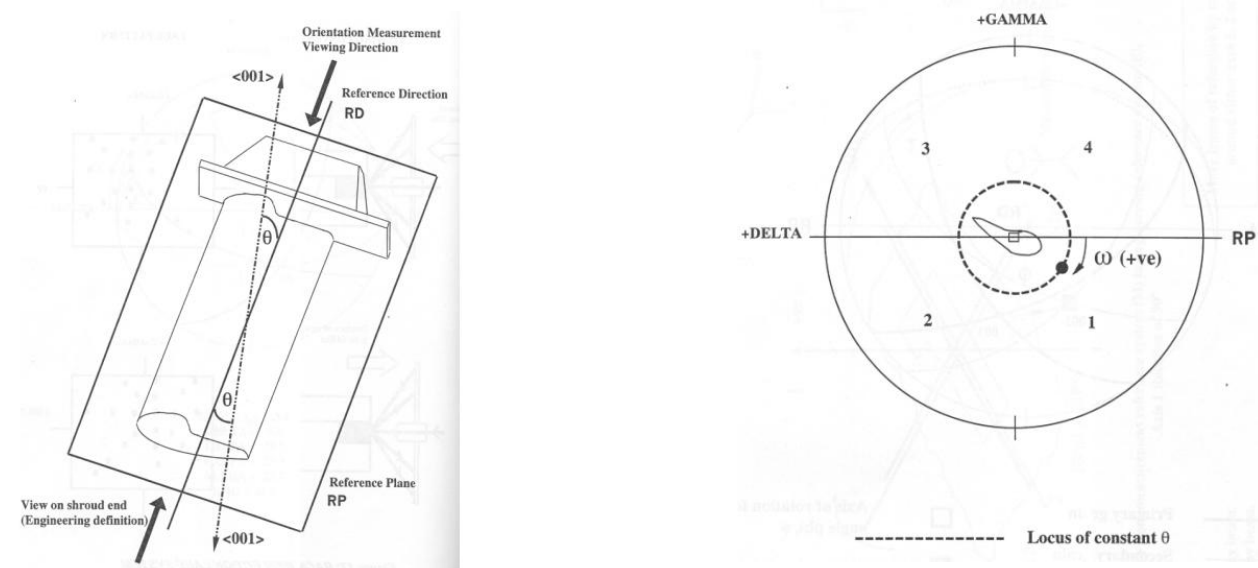

Figure 2 - Indication of blade reference axis Figure 3 - Indication of Omega relative to blade $\mathrm{X}$-Ray Diffraction is used to define the alignment of the crystal structure relative to the component reference system (usually the blade length axis). A constant static reference face is irradiated on each blade (Figure2), located normal to the reference axis of the blade, and from which angles (described by Cullity ${ }^{[10]}$ ) may be determined defining the crystal orientation of each blade. Theta $(\theta)$ is a combination of the angles $\gamma$ and $\delta$, and is the deviation from the reference direction to the nearest $<001>$ direction. Omega $(\omega)$, indicated in Figure 3 is the degree of rotation of the $<001>$ axis about the blade reference plane. 


\section{Production Technique}

The Investment Casting process is comprehensively described by Beeley \& Smart ${ }^{[11]}$. Directional solidification is achieved by the 'high speed withdrawal process' described by Paine ${ }^{[12]}$. Superheated molten alloy is cast into a bottomless ceramic mould attached to a water-cooled, retractable copper chill plate and surrounded by a radiant heat source which maintains both mould and alloy above the alloy liquidus temperature. The mould is vertically withdrawn at a pre-programmed rate from the radiant furnace zone. From the initial chill interface nucleation, grain growth occurs with elimination of unfavourable $<001>$ aligned grains in a starter block beneath the casting columnar, multi-crystalline structures are then developed in the casting. Single Crystal castings are achieved by incorporating a crystal selector beneath the component cavity above the chill plate and may take the form a constriction, a series of steps, a ramp or a $360^{\circ}$ helix. Such geometric shapes permit epitaxial columnar growth of the $<001>$ oriented grains whilst selectively eliminating all except one grain most suited for survival by its orientation with respect to the direction of heat flow ${ }^{[7]}$.

\section{Experimental}

A detailed survey of a standard production single crystal part was undertaken. Input variables and resulting product quality were monitored for a sample size of one year's worth of production data. A trial was undertaken in order to investigate the dependence of resulting product quality on a process variable. $\theta$ and $\omega$ angles were monitored for all standard production and trial blades. Grain structure measurements of dissected cast starter blocks were carried out on random samples of standard production and trial parts in order that direct comparisons could be made between the intentionally imposed variation and the base line standard process.

\section{- Standard Production Survey}

The proprietary single crystal part under observation had an imposed crystal orientation tolerance of $\cdot 20^{\circ} \theta$ of any $<001>$ pole from the blade reference axis, which lies along the length of the blade and no restrictions on secondary orientation or on the angle $\omega$. The standard process for this casting requires that patterns are assembled in clusters of ten in a radial formation above ten starter blocks protruding from a wax base plate equally spaced around a central downpole positioned normally to the base plate. The $360^{\circ}$ wax helix shaped grain selectors are adjoined to the tops of the starters at repeatable orientations equidistant from the downpole. The dimensions of the starter blocks permit a columnar grain growth of $25 \mathrm{~mm}$.

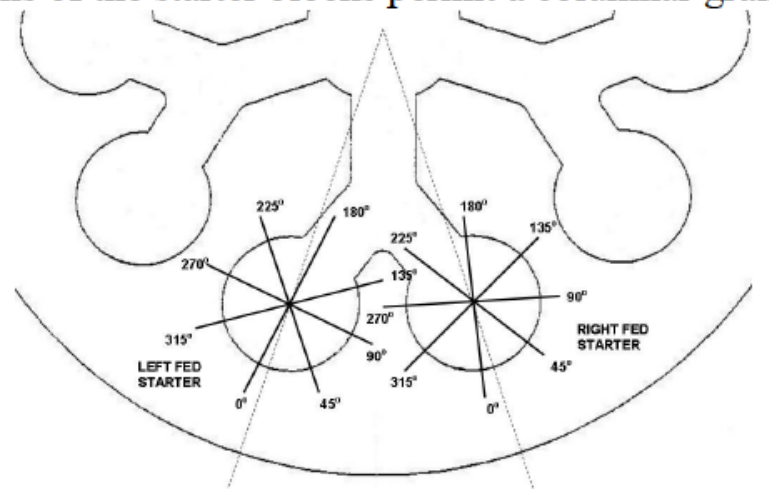

Figure 4-Section of base plate outlining base of Left and Right hand fed starters and corresponding omega scale

Patterns are oriented with the blade trailing edge towards the downpole and root end down such that the blade reference axis is parallel to the DS temperature gradient the mould will experience in the furnace. The casting is bottom-fed through the starter block and helixes via a gating system linked to the downpole (Figure 4). The system consists of five radial channels adjoined to the chill plate, each channel forking to supply two blades with alloy. The two types of starter block will be referred to hereafter as either left hand or right hand fed starters. Wax 
assemblies are then shell moulded by a 3-axis automated robot, in a proprietary shell system comprising two prime coats and a number of concrete coats. Moulds are dipped base down and drained at an angle of $30^{\circ}$ from the vertical whilst being rotated clockwise about the length axis of the mould. Moulds are cast at a pressure of $7 \times 10^{-6}$ bar and mould temperature of $1470^{\circ} \mathrm{C}$. Once cast and fully heat treated, each Single Crystal casting is assessed for crystal orientation measurement. During X-ray diffraction, each casting is located on datum points in a repeatable orientation such that crystal measurements are determined relative to the blade reference axis and that the angle $\omega$ can be translated back to the known orientation scale on the original wax assembly (Figure4).

\section{- Inverted Mould Trial}

The effect of drainage orientation on shell build up was investigated by inverting the wax assemblies on the robot and dipping and draining them base upwards in order to provoke slurry drainage away from the base of the assembly. Eight moulds were dipped in this manner then cast as per standard procedures.

\section{Results \& Discussion}

- Standard Production Survey

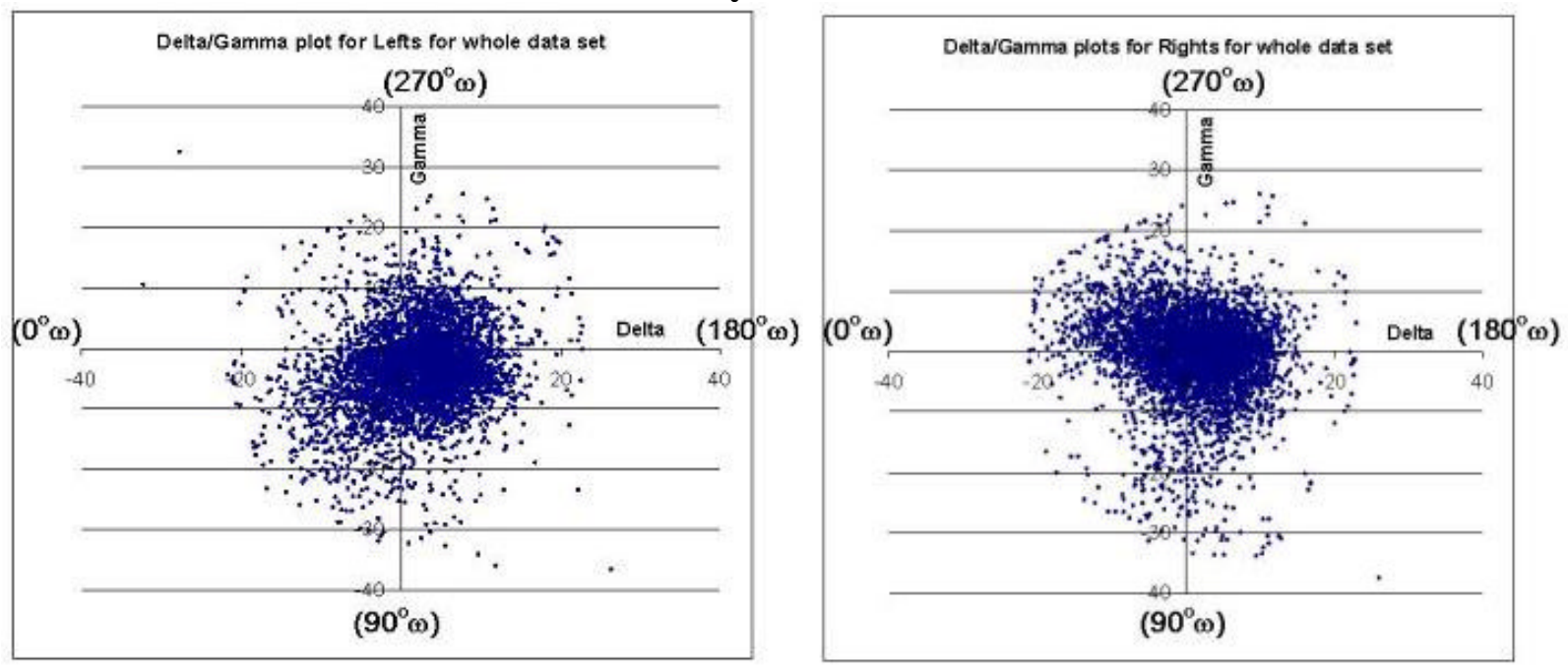

Figure 5 -Delta/Gamma plots for Left and Right type starter blocks

Figure 5 shows Delta/Gamma plots for the standard blades distinguishing whether the origin was from a Left or Right type starter block (for the $\omega$ scale indicated in Figure 4). It is clear that inherent asymmetries in the geometry of the wax dual fed starter system lead to resulting asymmetries in shell build up around the starter blocks and the consequential distribution of crystal orientations relative to the geometry of the blades. If the preferential growth was relative to location in the furnace, there would not be the distinction between Left and Right type starter blocks.

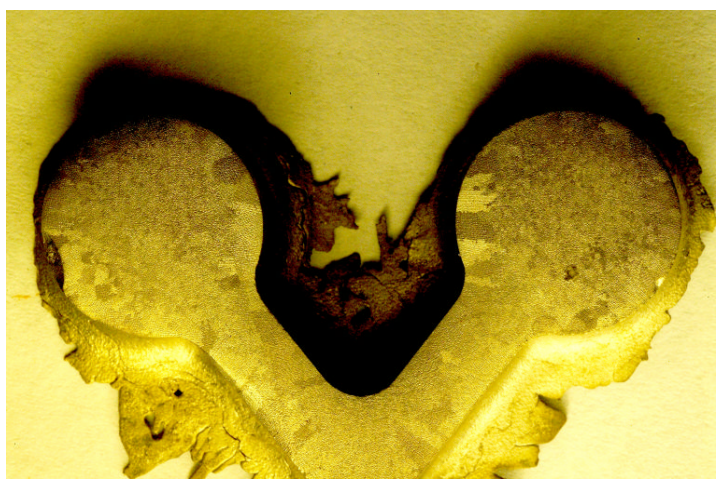

Figure 6 - Dissected and etched Standard Production Starter Pair

The etched grain structure of cast starter blocks was monitored at horizontal cross-sections mid-way up the length in order to determine the shape of the liquidus as it had progressed up 
the starter block. Figure 6 is an example of a dissected standard production starter block. The image of the etched pair of starter blocks displays a non-uniformly sized grain structure as the cross-section suggesting a curved liquidus as solidification progressed up the length of the starter block. This theory is supported by the fact that nucleated crystals whose $<001>$ axes are more favorably aligned with the thermal gradient will pinch off those less favorably aligned as their growth occurs up the starter block ${ }^{[9]}$. This would produce the preferentially oriented array of grains seen at the top of the starter that the helix can select from to produce the crystal orientations observed in Figure 5. Measurements of grain width were taken radially at the edge of the starter blocks, at repeatable $\omega$ values and averaged results for the standard production are shown in Figure 7. The results indicate an asymmetrical distribution of grain sizes (with Lefts reflecting Rights) which supports the theory of a curved liquidus and links to the asymmetrical crystal orientation distribution seen in the resulting blades.

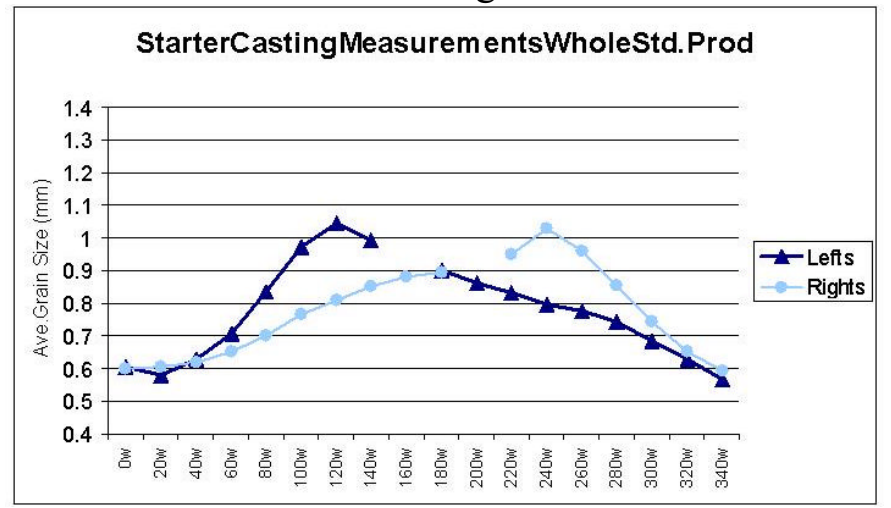

Figure 7 - Graph of Average Grain Size measurements at cross-section edge of dissected standard production starter blocks

\section{- Trial Results}

The inverted mould trials produced a thinner, more uniform shell coverage around the starter blocks. The resulting Delta/Gamma plot (Figure 8) when compared to the control moulds from the same dip run shows a tight distribution centred at the origin (nominal).

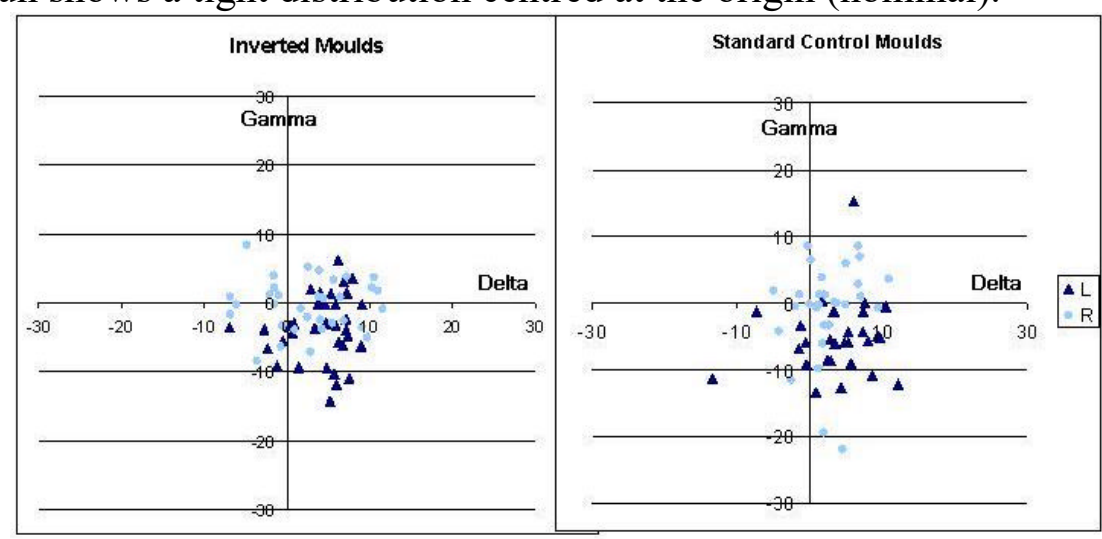

Figure 8-Delta/Gamma plots for Inverted and Control Moulds

This supports the theory that a more uniform, planar liquidus was achieved as it progressed up the starter blocks. Statistical analysis of the standard production and trial data confirms a more capable process for the trial data with a PPU of 0.96 on the upper limit of $20^{\circ} \theta$ compared to a PPU of 0.52 for the standard production data. The resulting starter block grain structure from the trial moulds was a fine, uniform grain size, across the section, right up to the edge (Figure 9). Figure 10 are the corresponding grain size measurements for the starter blocks from all the trial moulds. Although the sample size of data from the trial is insufficient to validate the process as being more in control of crystal orientation than the standard process, there is no doubt from the grain size measurements that the propensity for the trial process to produce an off-axis grain in any omega orientation has diminished. 


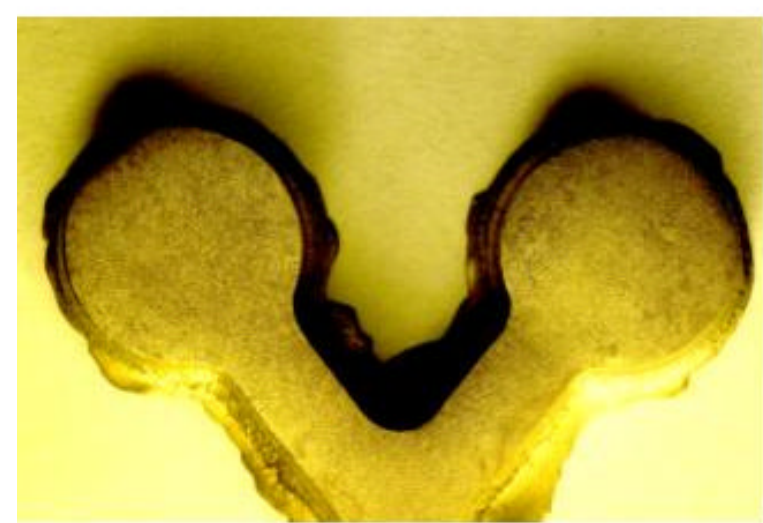

Figure 9 - Inverted Trial Mould Starters

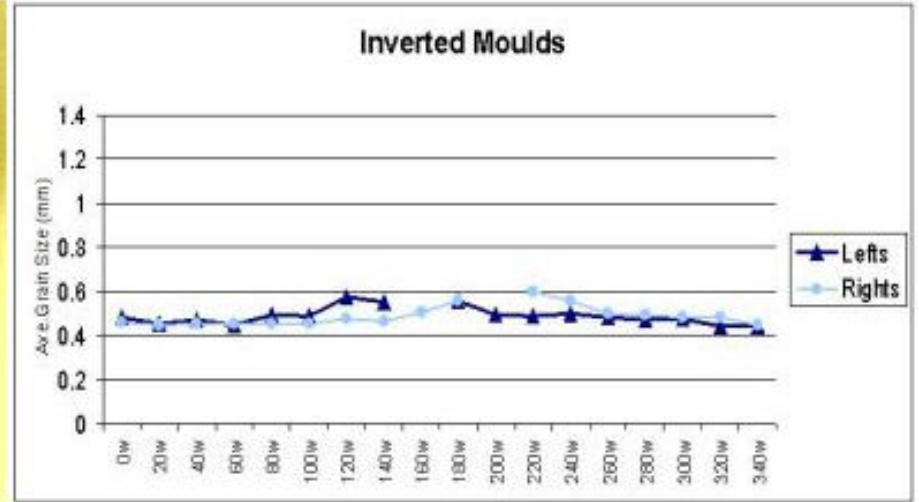

Figure 10 - Average Grain Size measurements

\section{Conclusions}

- The quality of crystals that reach the top of the starter block (i.e. the distribution of their deviations from the orientation of the blade reference axis) dictates the potential range of orientations of the crystal in the blade cavity. There is little potential for control of crystal selection, but there is however, potential for control over the quality of crystals that reach the top cross-section of the starter block.

- Any asymmetries in the uniformity of the temperature gradient will promote an increase in the mean deviation $\theta$, of the $<001>$ axes of crystals at the top of the starter block from their intended orientation in line with the blade axis.

- Variations in the shell thickness surrounding the starter blocks will have a direct influence over the thermal mass of shell available to supply heat to the molten alloy. This promotes asymmetries in the form and orientation of the liquidus as it progresses up the starter block and hence have a direct influence over which crystals will more favourably pinch off those less favourably aligned with the temperature gradient.

- The key to maximising single crystal production efficiency is the provision of uniform, thin coverage of shell around the starter blocks, achieved by the design of both the wax geometry and the shell dipping program, avoiding the promotion of sites for potential preferential shell build up.

\section{Acknowledgements}

The author would like to thank AETC Ltd. for the provision of data on single crystal production parts and the use of the casting facility where the standard production survey and trial described in this paper were carried out.

\section{References}

[1] Private communication with Dr. D.A.Ford

[2] A.Wagner, PhD Thesis, 2002, Imperial College, London

[3] R.Völk1, U.Glatzel and M.Feller-Kniepmeier, Acta Metallurgica, Vol 46, No. 12, p4395-4404,1998

[4] C.T.Sims, N.S.Stoloff and W.C.Hagel, 'Superalloys II High Temperature Materials for Aerospace and Industrial Power', Wiley Interscience Publication, New York, 1987

[5] M.Durand-Charre, 'The Microstructures of Superalloys', Gordon \& Breach Science Publishers, Amsterdam, 1997

[6] M.McLean, 'Directionally Solidified Materials for High Temperature Service', The Metals Society, Bristol,

UK, 1983

[7] TRW/DSSG/QUEST, 1980, p58-69

[8] P.Carter, D.C.Cox, C.A.Gandin and R.C.Reed, Materials Science \& Engineering A, Vol 280, p233-246, 2000

[9] M.H.Burden and J.D.Hunt, Crystal Growth, 1974, Vol 22, p109

[10] B.D.Cullity, 'Elements of X-Ray Diffraction', $2^{\text {nd }}$ Ed, Addison-Wesley Publishing Company Inc., USA, 1978

[11] P.R.Beeley and R.F.Smart, 'Investment Casting', Institute of Materials, London, 1995

[12] B.Paine, AETC Ltd., Paper No.17, p17.1-17.8 
